\title{
Comparison of the effect of various carbon fillers in the paraffin-based phase change materials------A Review
}

\author{
Peng Guo, Yuhan Du, Xianlin Zhang, Lingling Meng, Ting Zhang \\ Hongqi Street 626, Shijiazhuang 050091, China \\ guopeng.sci@gmail.com
}

\begin{abstract}
Keywords: paraffin-based phase change materials; expanded graphite; carbon fibers; carbon nanotubes; thermal conductivity; composite enthalpy.
\end{abstract}

Abstract. Using phase change material (PCM) as an energy storage medium is one of the most efficient ways of storing thermal energy. An important organic PCM is paraffin. There are a lot of advantages of paraffin, but an undesirable property of paraffin wax is its low thermal conductivity. The main methods to enhance the thermal conductivity of PCMs are metal particles, carbon fibers, expanded graphite (EG), and nanoparticles. Thus, this kind of PCM is heat transfer unstable and easy to layering. The addition of metal particles will lead to the increase of total weight of the system and cause uneven distribution of metal particles. So, we summarized and compared the researches with the effects of the addition of carbon fibers, EG and carbon nanoparticles to paraffin-based PCMs. We find that the peak value of the thermal conductivity of the composite PCM only appeared when the mass percentage of the carbon marterial in a appropriate location. Three-dimensional thermally conductive networks is better for the thermal conductivity enhancement. And molecular interaction between CNTs and the wax makes the composite enthalpy becomes higher than pure paraffin.

\section{Introduction}

Nowadays, thermal energy storage systems are crucial for reducing dependency on fossil fuels and also for minimizing $\mathrm{CO}_{2}$ emissions ${ }^{[1]}$. Thermal energy storage can be further classified into sensible heat storage, latent heat storage and thermo-chemical energy storage. Specifically, latent heat storage that is based on phase change materials (PCM) has received more attention than the others, due to its obvious advantages of high energy storage density and narrow temperature variation during the thermal energy charge/discharge process. PCMs adsorb/release heat during their phase-change process, and thus thermal energy storage/release is realized ${ }^{[2]}$.

An important organic PCM is paraffin, which is a kind of chain saturated alkane mixture, with general formula expressed by $\mathrm{C}_{\mathrm{n}} \mathrm{H}_{2 \mathrm{n}+2}$. There are a lot of advantages of paraffin, such as low cost, rich sources, colorless, stable physical, chemical properties, and the most important property of paraffin is larger phase change latent heat. An undesirable property of paraffin wax, however, is its relatively low thermal conductivity, which will become much lower in liquid and significantly decelerate the energy charging /discharging rates ${ }^{[3,4]}$. The coefficient of thermal conductivity of paraffin wax is around 0.2 $\mathrm{W} /(\mathrm{m} \cdot \mathrm{K})$, so its heat transfer efficiency is too low to make complete phase transition, meanwhile, it is too low to make the phase change latent heat fully released. And some reports showed that, improve the thermal conductivity and latent heat of PCMs is the main way to prolong module constant temperature of the $\mathrm{PCMs}^{[5]}$.

The main method to enhance the thermal conductivity of PCMs is to add some material of high thermal conductivity, such as metal particles, carbon fibers, expanded graphite (EG), and nanoparticles. The further researches on the enhancement of conductivity of PCM will focus on carbon fibers and nanoparticles. The addition of metal particles will lead to the increase of total weight of the system and cause uneven distribution of metal particles. Thus, this kind of PCM is heat transfer unstable and easy to layering. Therefore, future development of this kind of PCM is not optimistic. So, we summarized and compared the researches with the effects of the addition of carbon fibers, EG and 
carbon nanoparticles to paraffin-based PCMs. Summary of published experimental studies related to the effect of various carbon fillers in the paraffin-based PCMs is shown in Table 1

\section{Review of methods used for improving the thermal conductivity of paraffin wax}

\section{Dispersing EG}

EG as raw material is used in special workmanship. It is prepared from the natural flake graphite. It has fine properties of the natural flake graphite, such as good thermal conductivity, non-toxic. It also has fine properties which the natural flake graphite do not have, such as adsorption, porous network structure, the ecological environment of coordination and biocompatibility, etc. When paraffin play a role as PCMs and EG as additive, the composite PCM will get same new properties, such as higher thermal conductivity, form-stable as phase change process, a longer service life and excellent thermal stability ${ }^{[6]}$.

Xiao et al. ${ }^{[7]}$ prepared a shape stabilized PCM, which can keep the same shape in a solid state even the temperature is above the melting point of the paraffin by composing paraffin with a thermoplastic elastomer poly (styrene-butadiene-styrene). They got the cooling and heating curves of paraffin, composite PCM (EG mass ratio was 3 and $5 \%$ ). The thermal conductivity of the composite PCM with the EG mass ratio of $3 \%$ was increased more significantly.

Yin et al. preparaed a rapid thermal response composite PCM by absorbing paraffin into EG. The highest thermal conductivity of the composite samples can reach $4.676 \mathrm{~W} /(\mathrm{m} \cdot \mathrm{K})^{[8]}$. Sar1 et al. preparaed the paraffin/EG composites with the mass fraction of $2 \%, 4 \%, 7 \%$, and $10 \% \mathrm{EG}$, by absorbing liquid paraffin into the EG. The composite PCM with mass fraction of $10 \% \mathrm{EG}$ was considered as form-stable allowing no leakage of melted paraffin during the solid-liquid phase change due to capillary and surface tension forces of EG. Thermal conductivity of the pure paraffin and the composite PCM including 2, 4, 7 and $10 \mathrm{wt} \%$ EG were measured as $0.22,0.40,0.52,0.68$ and 0.82 $\mathrm{W} /(\mathrm{m} \cdot \mathrm{K})$, respectively ${ }^{[9]}$.

We found that the thermal conductivities of the composite PCM evidently improved compared to that of pure paraffin. But peak value of the thermal conductivity of the composite PCM only appeared when the mass percent of the EG in the appropriate location ${ }^{[7]}$. When the mass percent of EG was too much, the thermal conductivity of the composite PCM will be lower. The main reason is the existence of some lacunas and gases in the loose structure of the sample due to the high mass ratio of EG.

Dispersing carbon fibers

Carbon fiber is the carbon content of greater than $90 \%$ of the fibers collectively. It was developed a kind of new materials since 1960s. In the beginning, carbon fiber was only applied to traffic, industrial building and space. It has proportions as high coefficient of thermal conductivity (about $10 \sim 140$ $\mathrm{W} /(\mathrm{m} \cdot \mathrm{K}))$, small in size, high tension, high elasticity and low thermal expansion coefficient, etc. Therefore, carbon fiber has been gradually used to improve the performance of thermal conductivity of PCM.

Some types of enhancement techniques were studied. Such as the use of randomly oriented fibers, fiber brush, fiber cloth, carbon nanofiber (CNF) and so on. Jun et al. uses two carbon fibers to enhance the thermal conductivities of energy storage media. One is the technique using randomly oriented fibers, while the other is that of using a fiber brush. They find that both these types are useful for thermal conductivity enhancement and the brush is superior to the random one ${ }^{[10]}$.

Cui et al. studied thermal properties of CNF and carbon nanotube (CNT) filled PCM (soy wax and paraffin wax (PW) ). The composite PCM were prepared by the stirring of CNF or CNT in liquid wax at $60{ }^{\circ} \mathrm{C}$, with $\mathrm{CNF}$ and $\mathrm{CNT}$ doping levels of $1,2,5$, and $10 \mathrm{wt} \%$. The results showed that the thermal conductivity of composite PCM increases as CNF or CNT loading contents. Both CNF and CNT can 
improve the thermal conductivity of the composite, while CNF is shown to be more effective than CNT as the thermal conductive filler because of its better dispersion in thematrix ${ }^{[11]}$.

\section{Dispersing CNTs}

CNTs has the excellent properties like both EG and carbon fibers .Due to the properties such as low density, high thermal conductivity and adsorption, many research groups would like to add it to the paraffin wax. Experimental measurements showed that single coefficient of thermal conductivity of multi-walled CNTs as high as $3000 \mathrm{~W} /(\mathrm{m} \cdot \mathrm{K})$, far higher than that of conventional good thermal conductive materials ${ }^{[12]}$.

Some groups pointed out that because of the large specific surface area, CNT are prone to reunite deposits. After the deposition, thermal properties of the composites will be influenced. Thus, in the beginning, single walled CNT and multi-walled CNT were used as additive in the paraffin wax. At present, grafted CNT was concerned a lot. The existence of the branch reduces the trend to reunite deposits of grafted CNT and reduces the temperature of phase transformation point. Wang et al. use oleylamine and octanol as grafted onto the surfaces of the multi-walled CNT. The two kinds of grafted $\mathrm{CNT}$ and the pristine CNT were dispersed into the melting organic matrix to produce phase change composites. The composites with grafted CNT were proved homogenous and stable after repeating melting and solidification ${ }^{[13]}$. Wang et al. absorbing paraffin into $n$-octadecylamine-functionalized multi-walled CNTs. They found that comparing the influence of crude multi-walled CNTs and f-MWCNTs, it was obvious that $n$-octadecylamine-functionalized multi-walled CNTs showed a more positive effect on the thermal conductivity, which was attributed to better dispersibility of $n$-octadecylamine-functionalized multi-walled CNTs and utilization rate in the composite PCM ${ }^{[14]}$.

As grafted CNT are difficult to be prepared, some groups try to enhance the dispersibility of CNT in different solvents or polymers. A general approach for overcoming the agglomeration of CNT, improvement of their chemical reactivity, and the formation of a strong polymers /CNT interface is the use of functionalized CNT as filler into the polymer matrix ${ }^{[15]}$. For instance, Mallakpour et al. tried to treat CNT surface by acid, which made mixing of CNT with polar polymers. They preparaed alanine-based poly (amide-imide) / multi-welled CNT nanocomposites system ${ }^{[16]}$.

CNT are greatly concerned, not only because of its significantly higher thermal conductivity, but its molecular interaction between the wax. The addition of CNT can enhance latent energy of the composite PCM. Cao et al. preparaed a kind of composite. The composite is composed of paraffin wax infiltrated into a porous, deformable carbon nanotube sponge. The latter not only acts as a flexible encapsulation scaffold for wax but maintains a highly conductive network during the phase change process (for both solid and liquid states). They found that pure paraffin has an absolute enthalpy of $136.0 \mathrm{~J} / \mathrm{g}$. For PW/CNT composites, the apparent enthalpy (including the mass of nanotubes) increases almost linearly with increasing wax loading. At PW loadings of $<87 \mathrm{wt} \%$, the apparent enthalpy values are lower than that of pure PW due to a certain weight fraction of CNT contained in the composite. In spite of the mixture rule, their results show that above a critical loading of $91 \mathrm{wt} \%$, the composite enthalpy $(138.2 \mathrm{~J} / \mathrm{g})$ becomes higher than PW. This due to the wax-nanotube interaction 
Table 1 Summary of published experimental studies related to the effect of various carbon fillers in the paraffin-based phase change materials.

\begin{tabular}{|c|c|c|c|c|c|c|c|}
\hline \multirow[b]{2}{*}{ Author } & \multicolumn{3}{|c|}{ PCM used } & \multirow[b]{2}{*}{ Dispersed carbon materials } & \multicolumn{3}{|c|}{ Composited PCM } \\
\hline & Materials & $\begin{array}{l}\text { Thermal } \\
\text { conductivity ( W/ } \\
\mathrm{m} \cdot \mathrm{K} \text { ) }\end{array}$ & $\begin{array}{c}\text { Latent } \\
\text { heat }(\mathrm{J} / \mathrm{g})\end{array}$ & & $\begin{array}{c}\text { PCM : } \\
\text { Dispersed } \\
\text { carbon materials }\end{array}$ & $\begin{array}{c}\text { Thermal } \\
\text { conductivity ( W } \\
/ \mathrm{m} \bullet \mathrm{K})\end{array}$ & $\begin{array}{l}\text { Latent } \\
\text { heat }(\mathrm{J} / \mathrm{g})\end{array}$ \\
\hline Z. Zhang \& Fang, 2006 [18] & paraffin & & 188.69 & expanded graphite & $6: 1$ & & 161.45 \\
\hline $\begin{array}{l}\text { Yin, Gao, Ding, \& Zhang, } 2008 \\
\text { [8] }\end{array}$ & paraffin & 0.2697 & & expanded graphite & $15: 1$ & 4.676 & 189.0 \\
\hline Z. Zhang et al., 2012 [19] & paraffin & & 188.2 & expanded graphite & $11.5: 1$ & & 170.3 \\
\hline Kao, Li, Lv, \& Tan, 2012 [20] & $\begin{array}{l}\text { paraffin and organic } \\
\text { montmorillonite }\end{array}$ & & 37.56 & expanded graphite & $9.25: 1$ & & 112.21 \\
\hline Sarı \& Karaipekli, 2007 [9] & paraffin(n-docosane & 0.22 & 194.6 & expanded graphite & $9: 1$ & 0.82 & 178.3 \\
\hline Li \& Wu, 2011 [21] & $\begin{array}{l}\text { paraffin and } \\
\text { diatomite }\end{array}$ & & 109.37 & expanded graphite & $11.5: 1$ & & 108.13 \\
\hline Nakaso et al., 2008 [4] & packed paraffin & 0.21 & & carbon fiber cloths & & 0.42 & 0.58 \\
\hline Nakaso et al., 2008 [4] & packed paraffin & 0.21 & & carbon fiber brushes & & 0.75 & 0.58 \\
\hline $\begin{array}{l}\text { Fukai, Kanou, Kodama, \& } \\
\text { Miyatake, } 2000[10]\end{array}$ & paraffin & 0.26 & & Randomly oriented carbon fibers & & 0.5 & \\
\hline $\begin{array}{l}\text { Fukai, Kanou, Kodama, \& } \\
\text { Miyatake, } 2000[10]\end{array}$ & paraffin & 0.26 & & carbon fiber brush & & 0.5 & \\
\hline Cui, Liu, Hu, \& Yu, 2011 [11] & paraffin & 0.320 & & carbon nanofiber & & 0.450 & \\
\hline Wang, Xie, \& Xin, 2009 [22] & paraffin & & 165.3 & MWCNTs & $24.5: 1$ & & 163.8 \\
\hline Wang, Xie, \& Xin, 2011 [13] & paraffin & 0.22 & 142.2 & G18-graft- MWCNTs & & & 148.2 \\
\hline Wang, Xie, \& Xin, 2011 [13] & paraffin & 0.22 & 142.2 & G8-graft- MWCNTs & & & 139.1 \\
\hline Chen et al., 2012 [17] & paraffin & $0.16-0.20$ & 136.0 & carbon nanotube sponge & $\begin{array}{c}\text { paraffin } \\
\text { loadings }<87 \%\end{array}$ & & $<136.0$ \\
\hline Chen et al., 2012 [17] & paraffin & $0.16-0.20$ & 136.0 & carbon nanotube sponge & 10.11:1 & & 138.2 \\
\hline
\end{tabular}




\begin{tabular}{|c|c|c|c|c|c|c|c|}
\hline Chen et al., 2012 [17] & paraffin & $0.16-0.20$ & 136.0 & carbon nanotube sponge & $4: 1$ & 1.2 & \\
\hline Fang, X., et al.,2013 [23] & n-eicosane & 0.4079 & 262 & graphene nanoplatelets & $9: 1$ & 2 & 220 \\
\hline Cao et al., 2013 [24] & paraffin & 0.21 & & EG-CNT & $0.96: 1$ & 10.5 & \\
\hline Cao et al., 2013 [24] & paraffin & 0.21 & & EG-CNT & $0.75: 1$ & 6.5 & \\
\hline $\begin{array}{l}\text { Tang, Sun, Yu, \& Wang, } 2014 \\
{[14]}\end{array}$ & paraffin & 0.285 & 127.8 & MWCNTs & $19: 1$ & & 129.3 \\
\hline $\begin{array}{l}\text { Tang, Sun, Yu, \& Wang, } 2014 \\
{[14]}\end{array}$ & paraffin & 0.285 & 127.8 & $\begin{array}{c}\text { n-octadecylamine-functionalized } \\
\text {-MWCNTs }\end{array}$ & $99: 1$ & 0.369 & 138.6 \\
\hline $\begin{array}{l}\text { Tang, Sun, Yu, \& Wang, } 2014 \\
\text { [14] }\end{array}$ & paraffin & 0.285 & 127.8 & $\begin{array}{l}\text { n-octadecylamine-functionalized } \\
\text {-MWCNTs }\end{array}$ & $19: 1$ & 0.467 & 140.1 \\
\hline $\begin{array}{l}\text { Tang, Sun, Yu, \& Wang, } 2014 \\
\text { [14] }\end{array}$ & paraffin & 0.285 & 127.8 & $\begin{array}{l}\text { n-octadecylamine-functionalized } \\
\text {-MWCNTs }\end{array}$ & $9: 1$ & 0.532 & 122.1 \\
\hline
\end{tabular}




\section{Comparison of EG, carbon fibers and CNT}

Peak value of the thermal conductivity of the composite PCM
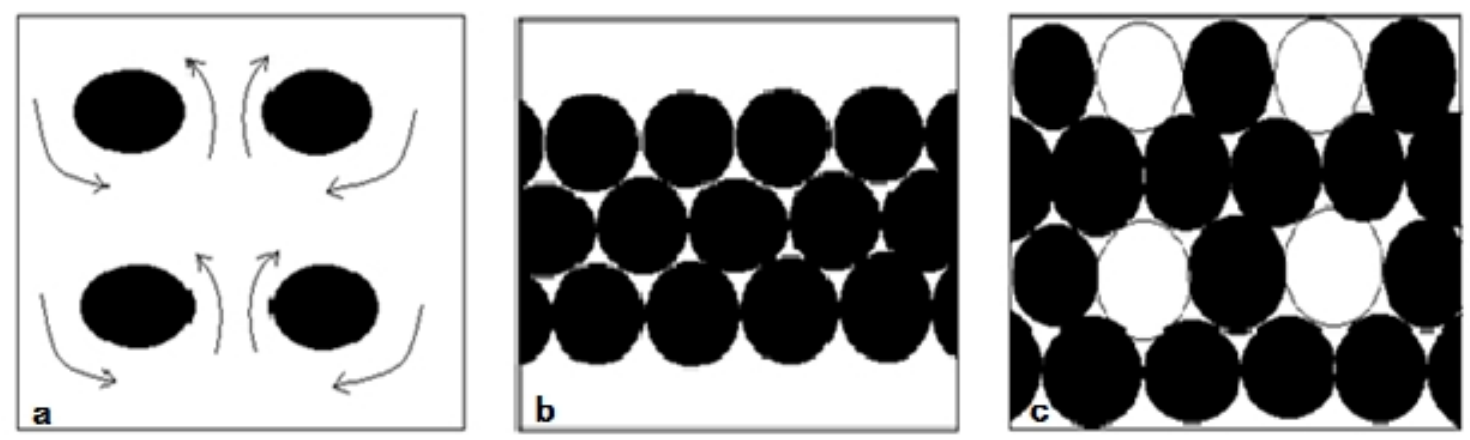

Fig 1. the mass percent of paraffin was too much(a), the mass percent of the carbon material in the appropriate location(b), the mass percent of carbon material was too much(c)

We found that the thermal conductivities of the composite PCMs improved evidently compared to that of pure paraffin, no matter what carbon material was introduced into paraffin. But peak value of the thermal conductivity of the composite PCM only appeared when the mass percentage of the carbon marterial in a appropriate location (Fig. 1 b). When the mass percentage of paraffin was too great, the particle of carbon material can not contact well. So the thermal conductivities mostly depend on the convection of liquid paraffin (Fig. 1 a). When the mass percentage of carbon marterial was too great, thermal conductivity of the composite PCM will be much lower. The main cause is the existence of some lacunas and gases in the loose structure of the sample, which may due to the high mass ratio of EG (Fig. $1 \mathrm{c})$.

Three-dimensional thermally conductive networks is better for the thermal conductivity enhancement.

EG has a three-dimensional plate-like nanostructure. Its volume expansion ratio is higher than that of the regular graphite. CNTs almost has a one-dimensional structure. But both CNTs and EG exhibited direction-dependent thermal conductivities, makes them difficult to be used as the thermal management materials with the aim of constructing three-dimensional thermally conductive networks in the composites.

Cao et al. introduced CNTs into EG. The EG-CNT nanocomposite is expected to be with excellent performance for heat transportation. In this way, neighboring graphite sheets were separated by numerous CNTs, which were rooted in and stabilized by the EG substrate, so the CNTs aggregation was also hindered. Paraffin was used as a matrix to embed EG-CNT nanocomposite and the thermal conductivity of the sample was higher than $10 \mathrm{~W} /(\mathrm{m} \cdot \mathrm{K})$, which indicated a promising candidate for thermal management in electronic package ${ }^{[24]}$.

Molecular interaction between CNTs and the wax makes the composite enthalpy becomes higher than pure paraffin

CNTs are greatly concerned, not only because of its significantly higher thermal conductivity, but also its molecular interaction between the wax. The addition of the EG tends to reduce the energy storage capacity of the composite PCMs due to the fact that they do not contribute to latent heat storage. Without CNTs, pure PW has an absolute enthalpy of about $136.0 \mathrm{~J} / \mathrm{g}$. Some results show that above a critical loading, the composite enthalpy becomes higher than pure paraffin ${ }^{[17]}$.

Shaikh et al. prepared three types of samples by doping shell wax with single-walled CNTs , multi-walled CNTs, and CNFs. The measured values of latent heat for all the samples showed a good enhancement over the latent heat of pure wax ${ }^{[25]}$. 
They used the theoretical model with experimental results for shell wax based nanocomposites and compared the the overall change in latent energy of the nanoparticle doped PCM sample. The components of the latent energy for the nanocomposite was the latent energy storage for the bulk PCM $\left(Q_{1}\right)$ and the latent energy due to molecular interaction between the nanoparticles and the wax $\left(Q_{m o l}\right)$. The latent energy for the nanocomposite is, thus,

$$
Q_{2}=Q_{1}+Q_{m o l}=m_{P C M} L+m_{C N T} \Delta H_{\max } \text {. }
$$

So $Q_{m o l}$ is the determiner of the overall change in latent energy of the nanoparticle doped PCM sample. They found the single-welled CNT have shown the foregrounded enhancement over the latent heat of pure wax. Because the single-welled CNT has the largest surface to volume ratio in the three CNTs, the molecular interaction between the single-welled CNT and the wax is higher ${ }^{[25]}$. The composite PCMs preparaed with EG or carbon fibers are not like this.

\section{Acknowledgements}

This work was supported by the Scientific Foundation for the Introduction of Researcher of Hebei College of Industry and Technology, China (No. BZ1101), Foundation on the science and technology of the University of Hebei Province, China (QN2015081) and the Scientific Foundation for Teachers of Hebei College of Industry and Technology, China (No. QZ-1303).

\section{References}

[1] I. Dincer, M. Rosen, Thermal energy storage: systems and applications, John Wiley \& Sons, 2002.

[2] C. Liu, F. Li, L.P. Ma, H.M. Cheng, Advanced materials for energy storage, Advanced Materials, 22 (2010) E28-E62.

[3] L.-W. Fan, X. Fang, X. Wang, Y. Zeng, Y.-Q. Xiao, Z.-T. Yu, X. Xu, Y.-C. Hu, K.-F. Cen, Effects of various carbon nanofillers on the thermal conductivity and energy storage properties of paraffin-based nanocomposite phase change materials, Applied Energy, 110 (2013) 163-172.

[4] K. Nakaso, H. Teshima, A. Yoshimura, S. Nogami, Y. Hamada, J. Fukai, Extension of heat transfer area using carbon fiber cloths in latent heat thermal energy storage tanks, Chemical Engineering and Processing: Process Intensification, 47 (2008) 879-885.

[5] Z-Q Guo, J-W Wu, Y-H Man, X-S Wu, Phase Change Analysis of PCM by ANSYS Finite

Element Method, New Technology \& New Process, 11(2007) 87-89.

[6] X-D Hu, X-N Gao, D-L Li, S-T Chen, Performance of paraffin/expanded graphite composite phase change materials. CIESC Journal, 64(2013) 3831-3837.

[7] M. Xiao, B. Feng, K. Gong, Preparation and performance of shape stabilized phase change thermal storage materials with high thermal conductivity, Energy conversion and management, 43 (2002) 103-108.

[8] H. Yin, X. Gao, J. Ding, Z. Zhang, Experimental research on heat transfer mechanism of heat sink with composite phase change materials, Energy Conversion and Management, 49 (2008) 1740-1746.

[9] A. Sar1, A. Karaipekli, Thermal conductivity and latent heat thermal energy storage characteristics of paraffin/expanded graphite composite as phase change material, Applied Thermal Engineering, 27 (2007) 1271-1277.

[10] J. Fukai, M. Kanou, Y. Kodama, O. Miyatake, Thermal conductivity enhancement of energy storage media using carbon fibers, Energy Conversion and Management, 41 (2000) 1543-1556.

[11] Y. Cui, C. Liu, S. Hu, X. Yu, The experimental exploration of carbon nanofiber and carbon nanotube additives on thermal behavior of phase change materials, Solar Energy Materials and Solar 
Cells, 95 (2011) 1208-1212.

[12] P. Kim, L. Shi, A. Majumdar, P. McEuen, Thermal transport measurements of individual multiwalled nanotubes, Physical review letters, 87 (2001) 215502.

[13] J. Wang, H. Xie, Z. Xin, Preparation and thermal properties of grafted CNTs composites, Journal of Materials Science \& Technology, 27 (2011) 233-238.

[14] Q. Tang, J. Sun, S. Yu, G. Wang, Improving thermal conductivity and decreasing supercooling of paraffin phase change materials by n-octadecylamine-functionalized multi-walled carbon nanotubes, RSC Advances, 4 (2014) 36584-36590.

[15] K.R. Reddy, B.C. Sin, C.H. Yoo, D. Sohn, Y. Lee, Coating of multiwalled carbon nanotubes with polymer nanospheres through microemulsion polymerization, Journal of colloid and interface science, 340 (2009) 160-165.

[16] S. Mallakpour, V. Behranvand, The influence of acid-treated multi-walled carbon nanotubes on the surface morphology and thermal properties of alanine-based poly (amide-imide)/MWCNT nanocomposites system, Colloid and Polymer Science, (2014) 1-7.

[17] L. Chen, R. Zou, W. Xia, Z. Liu, Y. Shang, J. Zhu, Y. Wang, J. Lin, D. Xia, A. Cao, Electro-and photodriven phase change composites based on wax-infiltrated carbon nanotube sponges, ACS nano, 6 (2012) 10884-10892.

[18] Z. Zhang, X. Fang, Study on paraffin/expanded graphite composite phase change thermal energy storage material, Energy Conversion and Management, 47 (2006) 303-310.

[19] Z. Zhang, N. Zhang, J. Peng, X. Fang, X. Gao, Y. Fang, Preparation and thermal energy storage properties of paraffin/expanded graphite composite phase change material, Applied Energy, 91 (2012) 426-431.

[20] H. Kao, M. Li, X. Lv, J. Tan, Preparation and thermal properties of expanded graphite/paraffin/organic montmorillonite composite phase change material, Journal of thermal analysis and calorimetry, 107 (2012) 299-303.

[21] M. Li, Z. Wu, Preparation and performance of highly conductive phase change materials prepared with paraffin, expanded graphite, and diatomite, International Journal of Green Energy, 8 (2011) 121-129.

[22] J. Wang, H. Xie, Z. Xin, Thermal properties of paraffin based composites containing multi-walled carbon nanotubes, Thermochimica Acta, 488 (2009) 39-42.

[23] X. Fang, L.-W. Fan, Q. Ding, X. Wang, X.-L. Yao, J.-F. Hou, Z.-T. Yu, G.-H. Cheng, Y.-C. Hu, K.-F. Cen, Increased thermal conductivity of eicosane-based composite phase change materials in the presence of graphene nanoplatelets, Energy \& Fuels, 27 (2013) 4041-4047.

[24] J. Cao, C. Men, H. Chen, G. Xu, Y. Zhang, Q. Li, In-situ fabrication of expanded graphite-carbon nanotube nanocomposite with enhanced thermal conductivity, Journal of Materials Science Research, 3 (2013) p50.

[25] S. Shaikh, K. Lafdi, K. Hallinan, Carbon nanoadditives to enhance latent energy storage of phase change materials, Journal of applied physics, 103 (2008) 094302. 